\title{
Modelo integrado de antecedentes y consecuencias del valor percibido por el egresado*
}

\author{
Sánchez Fernández, Raquel** \\ Iniesta Bonillo, Ma Ángeles*** \\ Cervera Taulet, Amparo**** \\ Schlesinger Díaz, Ma. Walesska*****
}

\section{Resumen}

Este artículo tiene como propósito construir y validar un modelo integrado de relaciones para el fomento de la creación de valor percibido por los egresados en el ámbito universitario. Con esta finalidad, se aplican las directrices del marketing de relaciones al análisis de la gestión educativa y al encuentro de servicio entre el profesor y sus estudiantes, mediante un estudio de campo realizado a 1000 egresados universitarios de dos universidades españolas. La técnica empleada fue el análisis factorial confirmatorio y el contraste del modelo estructural planteado a nivel teórico, con LISREL 8.80. Las conclusiones de este estudio suponen un avance en la literatura sobre educación superior, al corroborarse las relaciones planteadas, escasamente analizadas en este ámbito con anterioridad. Así, los resultados muestran que variables como la confianza, la calidad de la interacción alumnoprofesor, y la imagen de la Universidad influyen positivamente en la percepción del valor que ésta aporta, el cual, a su vez, afecta al nivel de satisfacción del egresado, contribuyendo a la formación de lealtad hacia la institución.

Palabras clave: Valor percibido, interacción, lealtad, universidad, marketing público.

Recibido: 22-01-11 Aceptado: 13-11-11

* Este trabajo ha sido realizado bajo el marco de los Proyectos de Investigación I+D SEJ2007-68105-C02-01/ECON y SEJ2007-68105-C02-02/ECON del Ministerio de Educación del Gobierno de España y con fondos ERDF.

** Profesora Contratada. Doctora de la Universidad de Almeria (España). e-mail: rasanche@ual.es

*** Profesora titular Universidad de Almeria (España).e-mail: miniesta@ual.es

**** Catedrática Universidad de Valencia. e-mail: cervera@uv.es

***** Profesora Facultad de Ciencias Económicas y Sociales de la Universidad del Zulia. e-mail: walesskas@gmail.com 
Modelo integrado de antecedentes y consecuencias del valor percibido

Sánchez F., R., Iniesta B., $M^{a}$ Á.; Cervera T., A. y Schlesinger D., Ma. W.

\title{
Integrated Model for Antecedents and Consequences of Value Perceived by Graduates
}

\begin{abstract}
The purpose of this article is to build and validate an integrated model of relationships for promoting value perceived by graduates in the university context. Relationship marketing principles are applied to the analysis of educational management and the service encounter between professor and students, through a field study of 1000 university graduates from two Spanish universities. The technique applied was confirmatory factor analysis and contrast of the proposed structural model on the theoretical level using LISREL 8.80. Conclusions of this study suppose an advance in the literature about higher education, corroborating the proposed relationships, which had hardly been analyzed before in this area. Results show how variables such as trust, the quality of student-professor interaction and university image influence positively perception of the value the university offers, which, in turn, affects graduate satisfaction and contributes to forming loyalty toward the institution.
\end{abstract}

Key words: Perceived value, interaction, loyalty, university, public marketing.

\section{Introducción}

El Consejo Europeo recoge en la denominada Estrategia de Lisboa que la Unión Europea debe convertirse en la más competitiva y dinámica economía mundial basada en el conocimiento, capaz de desarrollar un crecimiento económico sostenible con más y mejores empleos y mayor cohesión social. Este entorno plantea la necesidad de un enfoque más estratégico en la gestión de las instituciones educativas como centros de formación continua (Wong, 2005). Dichas instituciones desempeñan un rol sumamente importante en la formación de profesionales, así como en la creación, desarrollo, transferencia y adaptación científico-tecnológica, de modo que la labor llevada a cabo para responder adecuadamente a los requerimientos de la sociedad actual se constituye en un imperativo estratégico para el desarrollo de cada na- ción (Sebastián, 2004). En este contexto, los alumnos son descritos como clientes o stakeholders del sistema educativo (Chung y McLarney, 2000; Sakthivel y Raju, 2006), reconociéndose dicho sistema como generador de servicios centrado en el análisis y estudio de las expectativas y necesidades de sus clientes (Cheng y Tam, 1997).

La consideración de las instituciones de educación superior como generadoras de servicios para la compleja red de actores sociales (Cave et al., 1997; Sakthivel y Raju, 2006) frente a los cuales debe ofrecer respuestas eficaces y generar valor, determina la base para la aplicación del marco de la teoría del marketing de relaciones, cuya eficacia ha sido probada en organizaciones públicas y privadas, lucrativas y sin ánimo de lucro, consumidores finales (B2C) y empresas (B2B) (Arnett et al., 2003; Rapp, 2000; Stauss y Neuhaus, 1997). Desde este planteamiento, resulta fundamental el 
análisis del marco de relaciones de los alumnos como receptores de los servicios de educación ofrecidos por la Universidad (Oplatka y Hemsley-Brown, 2004), utilizando para ello enfoques estratégicos y de marketing que colaboren en el diagnóstico e implantación de programas formativos adecuados (Webster y Hammond, 2008).

El concepto de marketing de relaciones, que ha cobrado especial importancia en el ámbito de los servicios (HenningThurau et al., 2001), ha considerado variables tales como la confianza, el valor, el compromiso o la lealtad (Bryce, 2007; Gwinner et al., 1998; Morgan y Hunt, 1994; Parvatiyar y Sheth, 1994; Webster, 1994). Todas estas variables son necesarias para el desarrollo y mantenimiento de relaciones a largo plazo con los stakeholders. Sin embargo, la interacción entre los estudios en el ámbito de los servicios educativos y la línea de investigación en marketing de servicios es todavía muy escasa (Henning-Thurau et al., 2001; Javalgi et al., 2009), resultando ello en la escasez de aproximaciones de gestión de las relaciones en términos de teoría y de aplicaciones empíricas en este contexto. Frente al enfoque tradicional, surge la necesidad de establecer una estrategia centrada en reforzar el vínculo entre el público objetivo y las organizaciones, generando en el marco de esta relación un mayor valor tanto para los proveedores como para los clientes (Sánchez et al., 2001; Woodruff, 1997). La creación de valor se convierte así en una fuente potencial de ventaja competitiva en el sistema educativo (Sakthivel y Raju, 2006), destacándose la importancia de su impacto en el conocimiento y desarrollo personal del individuo. Tal y como destacan Webster y Hammond (2008), la filosofía que promulga la entrega de un valor superior a los clientes debe ser también aplicada a la gestión universitaria, en la medida en que se trata de organizaciones que poseen igualmente clientes, competencia, influencias externas, y que buscan alcanzar unos objetivos empresariales.

Esta investigación se plantea como una aproximación exploratoria a la vez que innovadora, ya que muestra la aplicación del marketing de relaciones al análisis de la gestión educativa y al encuentro de servicio entre el profesor y sus estudiantes. Más concretamente, su objetivo es construir y validar un modelo integrado de relaciones para el fomento de la creación de valor percibido por los egresados universitarios, conformado por diversas variables en la explicación del comportamiento de los jóvenes titulados superiores, como son la calidad de la interacción profesor-alumno, la confianza, la imagen de la Universidad, el valor percibido, su satisfacción y lealtad.

Para ello, y con el objeto de validar empíricamente el modelo de relaciones propuesto, se presenta un estudio empírico realizado sobre 1000 egresados universitarios de todas las titulaciones de dos universidades españolas, sobre los que se llevó a cabo un análisis factorial confirmatorio y el contraste del modelo estructural planteado a nivel teórico, con LISREL 8.80. Como paso previo se llevó a cabo una investigación cualitativa, esto permitió el desarrollo de un instrumento válido y confiable de medida, a través de escalas de medida Likert de 11 categorías (1-10). 
Modelo integrado de antecedentes y consecuencias del valor percibido Sánchez F., R., Iniesta B., $M^{a}$ Á.; Cervera T., A. y Schlesinger D., Ma. W.

La recogida de datos se llevó a cabo mediante un sistema de entrevistas telefónicas asistidas por ordenador (sistema CATI) mediante muestreo aleatorio simple utilizando el sistema SMS de aleatorización de la muestra. Del conjunto de la muestra, el $63,8 \%$ obtuvo el título en el 2005 y el $86 \%$ tiene intención de recibir cualquier tipo de formación en el futuro. El $77,4 \%$ se encuentra trabajando, el $16,8 \%$ está buscando trabajo y el 5,8\% restante ni busca ni trabaja. El 24,4\% tiene un salario entre 1200 y 1500 euros.

Se desarrolló una investigación cuantitativa empleando el cuestionario diseñado, realizándose primero un análisis factorial confirmatorio (AFC) mediante la metodología de modelización a través de ecuaciones estructurales, utilizándose el paquete estadístico LISREL 8.80. Ello permitió comprobar las propiedades psicométricas de las escalas utilizadas a través de la matriz de covarianzas y el método de estimación de máxima verosimilitud. Con posterioridad, se planteó un modelo estructural que permitió el contraste de las hipótesis planteadas.

De este modo, se pretende contribuir a la investigación desarrollada en torno a la relación cliente-empresa (en este caso, alumno-Universidad) (Bergami y Bagozzi, 2000; Bhattacharya y Sen, 2003), a través del análisis del proceso de creación de valor y sus principales antecedentes y consecuencias en este contexto de estudio (Baker et al., 2003). Así, es posible plantear algunas cuestiones de interés: ¿Cabe esperar diferencias en las relaciones entre las variables por el hecho de que el objeto de análisis sea la Universidad y no otro tipo de proveedor de servicios?, ¿Qué implicaciones para la gestión universitaria posee el análisis del paradigma relacional y la creación de valor?. Ciertamente, la identificación de variables útiles en la predicción y desarrollo de acciones de creación de valor en contextos universitarios resulta de gran interés para la articulación de planes de acción destinados a mejorar la actividad formativa de los colectivos de futuros titulados, garantizando el futuro de sus intereses profesionales a este respecto. Por ello, se afirma que del estudio pueden derivarse conclusiones importantes y directamente aplicables a la gestión de las instituciones de educación superior y a uno de sus más importantes stakeholders: los egresados.

\section{Determinantes y efectos del valor percibido en el contexto educativo}

En un entorno donde el conocimiento se contempla como un activo fundamental, los gobiernos han tomado conciencia de la importancia de desarrollar el capital intelectual de sus ciudadanos. Lo anterior implica la existencia de instituciones educativas capaces de diseñar e implantar programas formativos y metodologías docentes adecuadas que permitan configurarse en organizaciones de servicios innovadoras. En definitiva, como en otras organizaciones de servicio público, las instituciones de educación superior no pueden estar ajenas a este proceso, y requieren de la aplicación de estrategias de marketing (Webster y Hammond, 2008) y de una orientación al mercado u orientación al servicio de una sociedad que demanda esta educación (Flavián y Lozano, 2001; Webster y Hammond, 
2008). Tal y como se ha destacado en la literatura, la comprensión del comportamiento de los agentes implicados en la relación egresados-Universidad se nutre, entre otros elementos, del estudio de las fuentes generadoras de valor hacia los estudiantes (Sakthivel y Raju, 2006), consideradas como instrumentos estratégicos fundamentales que contribuyen al desarrollo intelectual, profesional y personal de los mismos.

Partiendo de la idea original de Porter (1985) que la ventaja competitiva de una organización proviene de su habilidad para la creación de valor, la relevancia que ha adquirido el valor como elemento clave en la gestión de servicios ha dado lugar a su consideración como un avance respecto a la calidad y a la satisfacción (Cronin et al., 2000; Woodruff, 1997), apuntándose a una idea de relevo de variables clave en la investigación del servicio (Gil et al., 2005). Esta orientación permitirá a la organización utilizar su conocimiento de este valor, entre otros, para (i) aumentar su competitividad en el mercado (Ruiz de Maya, 2001); (ii) asignar de forma adecuada sus recursos en el diseño y prestación de servicios (Cronin et al., 1997); (iii) mejorar los resultados obtenidos en el servicio (Cronin et al., 2000); (iv) como medio de diferenciación de la competencia (Chen y Dubinsky, 2003; Porter, 1985); (v) y para poder conocer y predecir el comportamiento de compra de los consumidores (Chen y Dubinsky, 2003; Day, 2002).

Pese a la manifiesta importancia del valor en la literatura de marketing ( $\mathrm{Hu}$ et al., 2009), y su destacada capacidad para analizar y predecir el comportamiento de los consumidores (Chen y Du- binsky, 2003; Huber et al., 2007; Pura, 2005), se puede observar que el creciente marco de conocimiento desarrollado en torno a este concepto se encuentra bastante fragmentado (Wang et al., 2004; Woodruff, 1997). Algunas corrientes de investigación se centran en la funcionalidad del valor percibido (Chang y Wildt, 1994; Dodds y Monroe, 1985; Zeithaml, 1988), siendo este paradigma cognitivo el que ha dominado la disciplina de marketing (Holbrook y Hirschman, 1982). En este marco, el valor es definido como "la valoración global que hace el consumidor de la utilidad de un producto sobre la base de las percepciones de lo que entrega y lo que recibe" (Zeithaml, 1988: 14). Por otra parte, otras corrientes han apelado a la consideración de la dualidad racional-emocional "presente en todo comportamiento humano" (Babin et al., 1994: 645), definiéndose el valor como "una experiencia interactiva, relativa y preferencial" (Holbrook y Corfman, 1985: 40). En esta línea, han surgido algunas propuestas multidimensionales de la naturaleza del valor (Babin et al., 1994; De Ruyter et al., 1997; Holbrook, 1999; Mathwick et al., 2001; Sheth et al., 1991; Sweeney y Soutar, 2001).

En particular, en el ámbito educativo se observa el escaso número de aproximaciones al análisis de la creación de valor hacia los estudiantes (e.g. Baker et al., 2002; Sakthivel y Raju, 2006; Unni, 2005), y más aún a su relación con otras variables. Siguiendo a Sakthivel y Raju (2006), el valor percibido en el ámbito educativo no está basado en la mera transmisión de conocimiento técnico o en el grado obtenido por el egresado, sino que es algo más, la posibilidad de perfeccionar las habilidades comu- 
Modelo integrado de antecedentes y consecuencias del valor percibido Sánchez F., R., Iniesta B., Má Á.; Cervera T., A. y Schlesinger D., Ma. W.

nicativas, interpersonales y de liderazgo, y de adquirir conocimientos sobre las últimas tendencias, de estar expuesto a un clima laboral, y de afrontar los diversos retos a los que se enfrenta todo individuo a lo largo de su vida.

Con objeto de generar un primer marco conceptual y de desarrollo del encuentro de servicio entre los egresados y la Universidad, se plantea un modelo de relaciones entre algunas de las variables más significativas en el proceso de creación de valor hacia el egresado (Diagrama 1). Esta aproximación integradora tiene en cuenta la teoría del marketing de relaciones, e incluye varios aspectos de relevancia en la investigación educativa y de servicios, considerando las características particulares de las instituciones de educación superior y su interacción con los egresados como principales clientes. A continuación, se justifica el modelo planteado a través del análisis de cada una de las hipótesis que subyacen en el mismo.

\subsection{Calidad de la interacción alumno-profesor en el encuentro de servicio}

La calidad de la interacción se concibe como un elemento clave en las relaciones de éxito (Lindgreen y Wynstra, 2005). Se define como el grado de adecuación de una relación para satisfacer las necesidades del cliente (HenningThurau y Klee, 1997). La calidad de la interacción es un constructo diferente de la calidad del producto o servicio. En este sentido, Crosby et al. (1990) señalan que la calidad de servicio es una condición necesaria pero no suficiente para la calidad de la relación, y Roberts et al. (2003), quienes también hacen esta distinción, consideran que es un mejor predictor de

\section{Diagrama 1 \\ Modelo Integrado de valor percibido: antecedentes y consecuencias.}

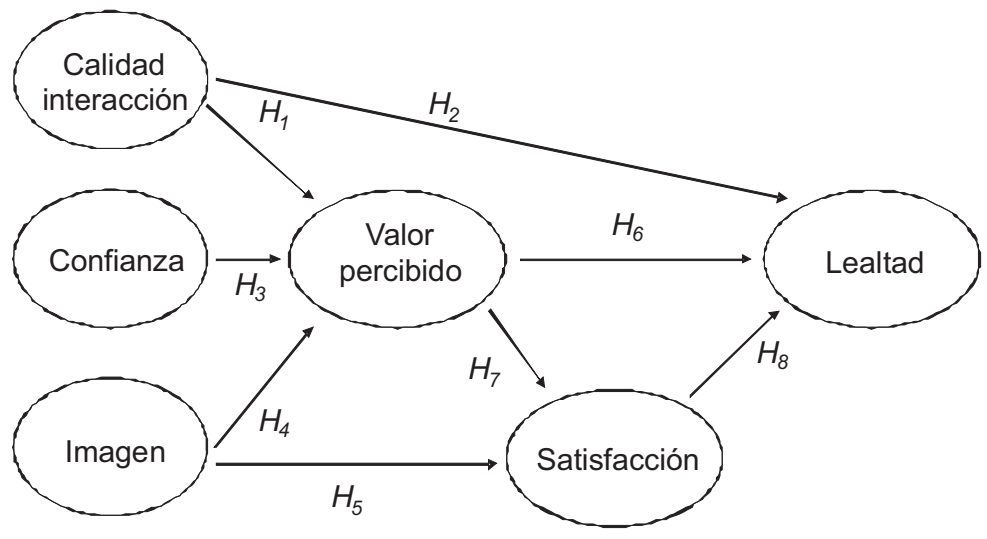

Fuente: Elaboración propia . 
las intenciones comportamentales que la calidad de servicio.

En el ámbito universitario, y en concreto en las relaciones alumno-universidad, cobra especial relevancia el estudio de la calidad de la interacción (Sakthivel y Raju, 2006). De acuerdo con Crosby et al. (1990), analizar la calidad de la interacción tiene sentido especialmente en el ámbito de los servicios, siendo de crítica importancia cuando el servicio es complejo, personalizado, y se realiza a lo largo de una serie de transacciones. A pesar de la importancia de esta temática, son escasos los trabajos que han estudiado la configuración de relaciones de calidad en el ámbito universitario (por ejemplo, Chung y McLarney, 2000; Venkatesh y Meamber, 2006), evidenciándose una laguna en esta línea de investigación. Los trabajos de Plewa et al. (2005) y Plewa y Quester (2006) serían algunos de los escasos ejemplos en este sentido, ya que analizan, el primero desde un enfoque cualitativo y el segundo cuantitativo, las relaciones universidad-industria, integrando variables del entorno organizacional, y variables que se refieren a la calidad de la interacción, como la confianza, el compromiso y la satisfacción. De este modo, el encuentro de servicio entre el alumno y el profesor es analizado como un tipo específico de servicio, donde la duración y la atención especial durante el encuentro constituyen factores significativos para la satisfacción de los egresados (Price et al., 1995).

Según Chung y McLearny (2000: 485), "la enseñanza es un encuentro de servicio", donde la reforma del proceso educativo requiere que el alumno sea tratado como el consumidor de un servicio (Krehbiel et al., 1997). En este sentido, es importante que el profesor considere al alumno como un partícipe activo del encuentro de servicio (Chung y McLarney, 2000). A partir de este planteamiento, es posible afirmar que el encuentro de servicio posee una especial influencia en el valor percibido (Keng et al., 2007). De allí que se plantee lo siguiente:

H1: La calidad de la interacción alumno-profesor influye en el valor percibido por el egresado.

Por otra parte, varios estudios señalan que las sensaciones experimentadas por los consumidores en su contacto con la organización determinan la formación de intenciones comportamentales futuras positivas hacia la misma (Mattila y Enz, 2002; Wong, 2004). De hecho, autores como Vesel y Zabkar (2010), Yavas y Babakus (2009) o Pollak (2009) analizan la relación entre la calidad de la interacción y la lealtad del consumidor en el comercio minorista, lo que resulta necesario tomar como base este tipo de investigaciones para determinar si dicha relación también se sostiene en el ámbito educativo. Por tanto, planteamos:

H2: La calidad de la interacción alumno-profesor influye en la lealtad del egresado hacia la institución.

\subsection{Confianza}

El interés de las empresas por captar nuevos clientes y retener a los actuales ha hecho de la búsqueda y análisis de los factores que motivan tales circunstancias un objetivo muy atractivo para investigadores y empresas. La confianza es uno de esos factores determinantes del éxito de las relaciones a largo plazo, habiéndose considerado como una variable 
Modelo integrado de antecedentes y consecuencias del valor percibido Sánchez F., R., Iniesta B., Má Á.; Cervera T., A. y Schlesinger D., Ma. W.

mediatizadora clave de los intercambios (Morgan y Hunt, 1994), pues su existencia promueve la eficiencia, productividad y efectividad de los resultados de la organización. Se entiende que "existe cuando una de las partes del intercambio tiene confianza en la fiabilidad e integridad de la otra parte" (Morgan y Hunt, 1994: 23). Si confianza y compromiso faltaran, la relación tendería a desaparecer (Wetzels et al., 1998). Estas afirmaciones son especialmente ciertas en el campo del marketing de servicios.

En el contexto educativo, y siguiendo la definición de Morgan y Hunt (1994), los alumnos podrán confiar en la integridad y fiabilidad de las instituciones de educación superior, la cual estará basada en las experiencias personales de los alumnos con los miembros de la comunidad universitaria (Henning-Thurau et al., 2001). Pocas investigaciones se han aproximado al estudio de la confianza en este ámbito. Cabe mencionar las de Henning-Thurau et al. (2001) y Holdford y White (1997), que han adaptado la teoría de la confianza-compromiso de Morgan y Hunt (1994) al contexto educativo para analizar la interacción alumno-universidad.

La literatura de servicios ha analizado la relación existente entre la confianza y el valor percibido (Lentz et al., 2004; Nijssen et al., 2003; Sirdeshmukh et al., 2002). Sirdeshmukh et al. (2002) afirman que la confianza crea valor al proporcionar beneficios relacionales derivados de la interacción entre la organización y el consumidor, reduciéndose así la incertidumbre entre los clientes (Morgan y Hunt, 1994). En este sentido, se propone lo siguiente:
H3: La confianza del egresado en la institución educativa influye en su percepción de valor.

\subsection{Imagen de la universidad}

Una imagen es un conjunto de notas adjetivas asociadas espontáneamente a un estímulo dado, el cual ha desencadenado previamente en los individuos una serie de asociaciones que forman un conjunto de conocimientos denominados creencias o estereotipos. Es la suma de creencias, ideas e impresiones que tiene una persona acerca de un objeto (Kotler y Fox, 1995).

La imagen percibida de las instituciones de educación superior juega un papel crítico en las actitudes de los públicos de dicha institución hacia la misma (Landrum et al., 1998). Ellas necesitan mantener o desarrollar una imagen distintiva con el objetivo de crear una ventaja competitiva en un mercado crecientemente competitivo (Paramewaran y Glowacka, 1995). Los diferentes públicos de las universidades extraen conclusiones acerca de la imagen general de una institución a partir de las impresiones que tienen acerca de sus fortalezas y debilidades (Ivy, 2001). Este estudio se centra en la percepción de los egresados.

La imagen institucional de la universidad tiene una importante incidencia en las decisiones de sus clientes, teniendo un efecto tanto en la retención de los estudiantes actuales como en la atracción de los potenciales (Helgesen y Nesset, 2007; Landrum et al., 1998), y en las actitudes de otros públicos que pueden afectar a su nivel de financiación (Landrum et al., 1998). Esta importancia 
de la imagen en el contexto universitario constituye un nuevo ámbito de interés como objeto de estudio y como ámbito de aplicaciones prácticas de la gestión del marketing (Beerli et al., 2002).

Precisamente desde la perspectiva de marketing, el impacto de la imagen corporativa en el comportamiento del consumidor ha sido reconocido a pesar de la falta de evidencia empírica. La relación entre la imagen corporativa y el valor percibido no ha sido objeto de especial atención en la literatura del ámbito educativo. Sin embargo, en otros contextos de estudio la imagen corporativa ha sido analizada como un antecedente o como mediadora de diversas variables relacionadas con la evaluación de las organizaciones, productos o servicios -calidad percibida, valor percibido, lealtad- individualmente o junto con la satisfacción (Beerli et al., 2002; Nguyen y LeBlanc, 2001; Pina et al., 2006). En particular, la relación imagen-valor ha sido validada en la literatura de servicio (Barich y Kotler, 1991; Nguyen y LeBlanc, 2001). Según lo anterior, se propone la siguiente hipótesis

H4: La imagen percibida de la universidad influye en la percepción de valor del egresado.

La influencia de la imagen corporativa sobre la satisfacción ha sido soportada empíricamente en la literatura (Andreassen y Lindestad, 1998; Beerli et al., 2002). Sin embargo, la influencia de la imagen en la satisfacción requiere de una evaluación más completa, puesto que también han sido observados algunos resultados contradictorios. Por ejemplo, Bloemer et al. (1998) no validan la hipótesis de que la imagen, a través de la satisfacción, posee un efecto indirecto sobre la lealtad, mientras que en el trabajo de Bloemer y De Ruyter (1998) se demostró lo contrario. A la vista de esta controversia, y con objeto de analizar esta relación en el ámbito educativo, se plantea lo siguiente:

H5: La imagen percibida de la universidad influye en la satisfacción del egresado.

\subsection{Satisfacción y lealtad del egresado}

La importancia de medir la variable satisfacción reside, entre otras razones, en su relación con la lealtad del cliente (Söderlund, 2006). En el ámbito empresarial actual, caracterizado por la elevada competitividad existente, la repetición de la compra constituye un fenómeno necesario en orden a garantizar la supervivencia de las organizaciones, lo que dará lugar a la retención de clientes. Esta situación afecta a numerosos sectores, entre ellos las instituciones de educación superior (Helgesen y Nesset, 2007).

La satisfacción del consumidor ha sido ampliamente estudiada en la literatura, aunque no existe consenso acerca su naturaleza en el ámbito de los servicios, especialmente en educación superior (Helgesen y Nesset, 2007). Una de las adaptaciones realizadas a este contexto es aquella propuesta por Elliot y Healy (2001), que indican que se trata una actitud a corto plazo que resulta de la evaluación de la experiencia con el servicio educativo recibido.

La satisfacción ha sido uno de los conceptos que mayor polémica ha suscitado al estudiar su relación con el valor, por cuanto que se ha especulado acerca 
Modelo integrado de antecedentes y consecuencias del valor percibido Sánchez F., R., Iniesta B., $M^{a}$ Á.; Cervera T., A. y Schlesinger D., Ma. W.

de la naturaleza exacta de la misma. Según Woodruff y Gardial (1996: 86) "definir la distinción (y el vínculo) entre el valor para el cliente y la satisfacción del cliente es algo crítico debido a la natural afinidad entre los dos conceptos". La satisfacción y el valor para el consumidor son conceptos que claramente comparten algunas características comunes, aunque se trate de conceptos diferentes. Así, la evaluación post-compra de la satisfacción contrasta con el carácter dinámico del valor, el cual puede manifestarse en varias etapas del proceso de compra (Woodruff, 1997). Además, bajo la satisfacción subyace una orientación táctica que proporciona guías de actuación para mejorar los productos actuales, mientras que la orientación estratégica del valor proporciona mecanismos de actuación futura (Eggert y Ulaga, 2002).

Sobre la relación valor-satisfacción, se han llevado a cabo multitud de estudios que no siempre coinciden en sus conclusiones. En principio, en gran parte de la literatura se señala la existencia de una influencia positiva del valor percibido por el consumidor sobre la satisfacción (por ejemplo, Cronin et al., 2000; Tam, 2004; Varki y Colgate, 2001). Frente a estos estudios, otros rechazan la hipótesis de una posible relación (Andreassen y Lindestad, 1998), o defienden que la satisfacción del consumidor es un antecedente del valor percibido (Bolton y Drew, 1991; Petrick et al., 2001), que el valor sólo posee un efecto parcial en la satisfacción pero a través de su interacción con la calidad (Caruana et al., 2000), o que la satisfacción genera valor de consumo, el cual a su vez deviene en satisfacción basada en el valor (Oliver, 1999).
Dado que todavía no existe una opinión generalizada acerca de la relación valorsatisfacción, y que ha sido calificada de confusa (Eggert y Ulaga, 2002), especulativa (Day, 2002), y sujeta a cierta controversia (Martín et al., 2004), se formula la siguiente hipótesis en el contexto educativo, tomando como base los trabajos de Chen y Dubinsky (2003), Sakthivel y Raju (2006) y Yang y Peterson (2004):

H6: El valor percibido por el egresado influye en la satisfacción del mismo.

Por otra parte, la lealtad del egresado ha sido considerada como un aspecto esencial para la supervivencia de las instituciones de educación superior (Helgesen y Nesset, 2007). El mantenimiento de relaciones a largo plazo con los egresados puede generar ventajas competitivas para las Universidades (Henning-Thurau et al., 2001). La situación actual, caracterizada por la disminución del número de alumnos, el incremento de la tasa de abandono de estudios, y los requerimientos de la Declaración de Bolonia (1999), entre otros, justifican la importancia del análisis de la lealtad en este contexto (Helgesen y Nesset, 2007).

A pesar que el vínculo entre el valor y la lealtad ha sido reconocido a nivel teórico y soportado empíricamente (Cronin et al., 2000; Sirdeshmukh et al., 2002; Yang y Peterson, 2004), también se ha afirmado que la relación directa entre el valor percibido y la lealtad no está tan clara (Tam, 2004), no validándose esta relación (Patterson y Spreng, 1997; Varki y Colgate, 2001; Wang et al., 2004). En definitiva, se considera de interés determinar el poder explicativo que posee el valor percibido sobre la lealtad en el contexto 
de la educación superior mediante la siguiente hipótesis:

H7: El valor percibido por el egresado influye en la lealtad del mismo.

Asimismo, otros estudios han demostrado la influencia indirecta del valor percibido sobre la lealtad a través de la satisfacción del consumidor (Yang y Peterson, 2004), si bien otros no la soportan (Andreassen y Lindestad, 1998). Más aún, en el ámbito de los servicios en general, la satisfacción y la lealtad son considerados conceptos relacionados, siendo la satisfacción un antecedente de la lealtad (Dick y Basu, 1994). De forma similar, en el contexto de las instituciones de educación superior, los conceptos de satisfacción y lealtad han sido vinculados a través de su relación causal (Helgesen y Nesset, 2007; Söderlund, 2006). Por ello, se plantea lo siguiente:

H8: La satisfacción del egresado influye en la lealtad del mismo.

\section{Contrastación del modelo integrado de valor percibido en el ámbito universitario}

Tras haber especificado las características metodológicas de la investigación a realizar, caracterizado teóricamente las variables incluidas en el modelo y planteadas las hipótesis, se procede a exponer los resultados obtenidos con relación al objetivo planteado en este artículo.

Como punto de partida, considerando las respuestas obtenidas a la batería de ítems que componen el cuestionario de la investigación, el cálculo de las correlaciones ítem-total de forma aislada para cada componente permitió comprobar que todas las variables observadas presentaban un valor no inferior a 0,35 (Saxe y Weitz, 1982). Tras este paso previo, se llevó a cabo un análisis factorial confirmatorio con objeto de profundizar en el proceso de depuración de las escalas y evaluar sus propiedades psicométricas (Tabla 1). A pesar de que todos los ítems cargaron en su concepto de forma significativa $(p<0,01)$, los índices de modificación y los residuos estandarizados mostraron la conveniencia de eliminar un ítem de la escala de calidad de la interacción que hacía referencia a la buena relación entre profesores y alumnos "Cl3", otro de la variable confianza que reflejaba si la Universidad es percibida como institución sincera y honrada "C1" , otro de valor percibido que se refería al valor percibido al cursar los estudios considerando el tiempo, dinero y esfuerzo empleados "VP1", y finalmente otro de la escala de lealtad que reflejaba si el egresado volvería a elegir la Universidad para sus estudios "L1" con objeto de mejorar la bondad del ajuste planteado.

La bondad de ajuste del modelo de medida final se analizó a través de varios indicadores. Como puede observarse en la Tabla 1, los índices de bondad indican la existencia de un ajuste adecuado. El índice de ajuste comparativo (CFI), el índice de Tucker-Lewis o de ajuste no normado (NNFI), y el índice de ajuste incremental (IFI) presentaron valores superiores al recomendado de 0,90. Además, el valor del error de aproximación cuadrático medio (RMSEA) se sitúa dentro del límite máximo recomendado de 0,08 . El estadístico Chi-cuadrado resulta significativo, si bien esto puede deberse a la 
Modelo integrado de antecedentes y consecuencias del valor percibido Sánchez F., R., Iniesta B., $M^{a}$ Á.; Cervera T., A. y Schlesinger D., Ma. W.

Tabla 1

Resultados del análisis factorial confirmatorio final y fiabilidad de las escalas $(n=1000)$.

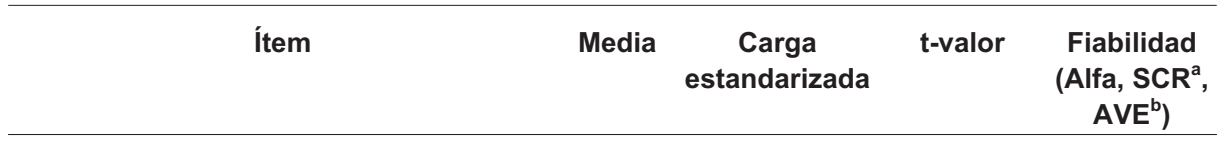

Calidad de la interacción

Alumno-Profesor (Peiró et al., 2005)

En general, los profesores con los que

6,91

1,38

40,76

Alfa $=0,93$

tenías contacto se mostraron interesados en

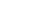

$\mathrm{SCR}=0,93$

enseñar a sus alumnos.

$\mathrm{AVE}=0,82$

En general, los profesores con los que

6,76

1,37

38,89

tenías contacto mostraron interés especial

en dar más que lo estrictamente académico.

En general, había una relación cercana

6,84

1,19

31,36

entre alumnos y profesores.

Media total cargas

Confianza (Morgan y Hunt, 1994)

La Universidad $X$ tiene una elevada integridad (rectitud/honorabilidad).

\begin{tabular}{|c|c|c|c|}
\hline 6,75 & 1,73 & 38,73 & Alfa $=0,97$ \\
\hline & & & $\begin{array}{l}\mathrm{SCR}=0,91 \\
\mathrm{AVE}=0,91\end{array}$ \\
\hline 6,83 & 1,81 & 41,00 & \\
\hline
\end{tabular}

Universidad X.

Se puede contar con que la Universidad $X$

6,79

1,84

43,07

va a actuar de forma correcta.

6,79

Media total cargas

Imagen (Nguyen y LeBlanc,2001)

Tienes una buena impresión de la

7,34

1,62

42,34

Alfa $=0,92$

Universidad X.

En tu opinión, la Universidad $X$ tiene una buena imagen entre sus usuarios.

7,23

1,34

36,11

$\mathrm{SCR}=0,92$

$\mathrm{AVE}=0,76$
Crees que la Universidad $X$ tiene mejor

6,64

1,24

24,44

En general, tienes una imagen positiva de la 7,28 Universidad X. 
Tabla 1

Resultados del análisis factorial confirmatorio final y fiabilidad de las escalas $(n=1000)$ (Continuación).

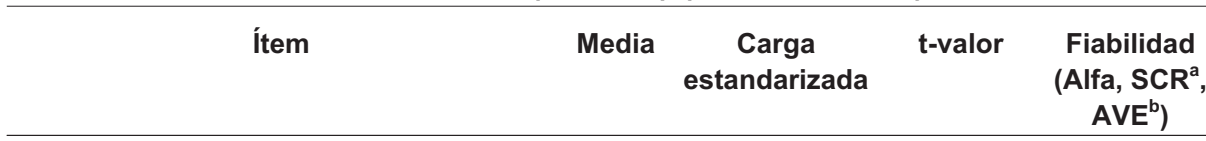

Valor percibido

(adaptada Dodds et al.,1991)

Teniendo en cuenta el tiempo, esfuerzo y dinero empleados, consideras que el valor recibido al cursar tus estudios es adecuado.

Comparando todo lo que has obtenido durante los estudios cursados (formación, servicio, ambiente, relaciones, entre otros) con todos los sacrificios realizados para obtener el título (tiempo y esfuerzo dedicados, dinero, entre otros), tu valoración de estos estudios es positiva.

El haber cursado estos estudios tiene valor para tu carrera profesional.

Media total cargas

6,52

2,04

37,26

Alfa $=0,86$

$\mathrm{SCR}=0,86$

$\mathrm{AVE}=0,69$

6,70

1,83

36,77

Satisfacción (Fornell, 1992)

Tu decisión de haber seleccionado la Universidad X fue acertada.

Tu experiencia en la Universidad $X$ ha satisfecho las expectativas que tenías.

En general, estás satisfecho con tu decisión de haber seleccionado la Universidad X para cursar tus estudios.

Media total cargas

Lealtad (adaptada Henning-Thurau et al., 2001 y Martensen et al., 1999).

Si tuvieras que realizar otros cursos, conferencias o estudios de mejora profesional seguramente considerarías a la Universidad X como primera opción.

Si alguien te pide consejo recomendarías la Universidad X.

7,78

1,91

41,40

Si se presentara la oportunidad comentarías

7,73

1,88

41,89 con tus amigos y familiares cosas positivas de la Universidad X. 
Modelo integrado de antecedentes y consecuencias del valor percibido Sánchez F., R., Iniesta B., $M^{a}$ Á.; Cervera T., A. y Schlesinger D., Ma. W.

Tabla 1

Resultados del análisis factorial confirmatorio final y fiabilidad de las escalas ( $n=1000)$ (Continuación).

\begin{tabular}{|c|c|c|c|c|}
\hline Ítem & Media & $\begin{array}{c}\text { Carga } \\
\text { estandarizada }\end{array}$ & t-valor & $\begin{array}{c}\text { Fiabilidad } \\
\text { (Alfa, SCR }^{\mathrm{a}}, \\
\left.\text { AVE }^{\mathrm{b}}\right)\end{array}$ \\
\hline $\begin{array}{l}\text { Animarías a familiares y amigos a que } \\
\text { estudiasen en esta Universidad. }\end{array}$ & 7,74 & 1,91 & 43,51 & \\
\hline Media total cargas & \multicolumn{4}{|l|}{7,68} \\
\hline Bondad de ajuste & \multicolumn{4}{|c|}{$X^{2}(155)=641,49, p<0,001$} \\
\hline \multicolumn{5}{|c|}{ a'SCR: Scale composite reliability o fiabilidad compuesta: $\rho_{c}=\left(\sum \lambda_{i}\right)^{2} /\left(\sum \lambda_{i}\right)^{2}+\sum \operatorname{Var}\left(\delta_{i}\right)$} \\
\hline \multicolumn{5}{|c|}{${ }^{\mathrm{b}} \mathrm{AVE}$ : Average variance extracted o varianza extraída: $A V E=\sum \lambda_{i}^{2} / \sum \lambda_{i}^{2}+\sum \operatorname{Var}\left(\delta_{i}\right)$} \\
\hline
\end{tabular}

Fuente: Elaboración propia.

alta sensibilidad de este indicador respecto al tamaño muestral (Bagozzi y Yi, 1988). Finalmente se observa que los coeficientes lambda estandarizados son significativos $(p<0,01)$, y los factores latentes están correlacionados de forma positiva.

Tras el análisis de la bondad del ajuste del modelo, se analizaron la fiabilidad y la validez de las escalas desarrolladas para las variables del modelo planteado. De este modo, tanto el coeficiente Alfa de Cronbach como los índices de fiabilidad compuesta (SCR) y varianza media extraída (AVE) dan muestra de la fiabilidad de las escalas desarrolladas ( $\mathrm{Ta}$ bla 1), pues los valores alcanzados son superiores al mínimo recomendado de 0,7 para Alfa (Nunnally, 1978), 0,6 para la fiabilidad compuesta (Bagozzi et al., 1992) y 0,5 para la varianza media extraída (Fornell y Larcker, 1981). Respecto a la validez de contenido, se asume que las escalas obtenidas miden los conceptos citados y no otros, ya que para su elabo- ración se llevó a cabo una extensa revisión de la literatura en esta materia, así como un estudio cualitativo previo. Por lo que se refiere a la validez convergente, puede observarse que los coeficientes estandarizados de cada indicador son significativos, es decir, que estos coeficientes son mayores que dos veces su error estándar (Anderson y Gerbing, 1988). El cumplimiento de esta condición se constata directamente ante la presencia de ponderaciones de las variables elevadas y significativas (valor de la t superior a 1,96) (Tabla 1). Por su parte, la validez discriminante queda comprobada a través de los métodos proporcionados por Anderson y Gerbing (1988). Siguiendo el primero de ellos, el intervalo de confianza ( \pm 2 veces el error estándar) de la correlación estimada entre dos de las variables consideradas $(\Phi)$ no incluía el valor uno. Adicionalmente, mediante el segundo método, se comparó la bondad del ajuste $\left(\mathrm{X}^{2}\right)$ de dos modelos de medición, donde en uno de ellos quedaba restringi- 
do al valor 1 la estimación del parámetro de correlación entre cada par de variables latentes. La significatividad del test demostró de nuevo la existencia de validez discriminante entre los componentes del valor. Finalmente, el test de la varianza media extraída también resultó positivo, ya que la correlación al cuadrado entre las dimensiones era inferior a las varianzas extraídas.

Posteriormente, se planteó un modelo de ecuaciones estructurales para el contraste del modelo de relaciones propuesto (Diagrama 1). Se observa que los índices de bondad de ajuste del modelo global son muy aceptables (Tabla 2), lo que sugiere que la red nomológica de relaciones se ajusta a los datos obtenidos en el estudio.

Asimismo, en cuanto a las hipótesis planteadas, los resultados demues- tran, por una parte, que la calidad de la interacción alumno-profesor influye positivamente en la percepción de valor que poseen los egresados sobre la experiencia de servicio en la universidad, siendo también positiva la relación existente entre la calidad de la interacción y la lealtad del egresado a la universidad. Por tanto, las hipótesis $\mathrm{H} 1$ y $\mathrm{H} 2$ se soportan. Además, los datos confirman la influencia positiva entre la confianza del egresado y su imagen percibida de la universidad hacia el valor percibido por el mismo, soportándose las hipótesis $\mathrm{H} 3$ y $\mathrm{H} 4$.

Por otra parte, los resultados indican que tanto la imagen percibida por el egresado como su valor percibido contribuyen a la satisfacción del mismo, de manera que las hipótesis $\mathrm{H} 5$ y $\mathrm{H} 6$ se confirman con los datos obtenidos. Más aún, se observa que, además de la satisfacción,

Tabla 2

Contraste del modelo de relaciones.

\begin{tabular}{|c|c|c|c|c|c|}
\hline \multirow[t]{2}{*}{ Enlaces en el modelo } & \multirow[t]{2}{*}{ Hipótesis } & \multicolumn{3}{|c|}{$\begin{array}{c}\text { Estimación de parámetros } \\
\text { estandarizados }\end{array}$} & \multirow{2}{*}{$\begin{array}{c}\text { Contraste } \\
\text { de } \\
\text { hipótesis }\end{array}$} \\
\hline & & Parámetro & Estimación & t-valor & \\
\hline Calidad interac. $\rightarrow$ Valor percibido & $\mathrm{H}_{1}$ & $\mathrm{Y}_{11}$ & 0,49 & $6,81^{* * *}$ & Aceptada \\
\hline Calidad interac. $\rightarrow$ Lealtad & $\mathrm{H}_{2}$ & $\mathrm{Y}_{31}$ & 0,37 & $6,52^{* * *}$ & Aceptada \\
\hline Confianza $\rightarrow$ Valor percibido & $\mathrm{H}_{3}$ & $\mathrm{Y}_{12}$ & 0,37 & $4,66^{* * *}$ & Aceptada \\
\hline Imagen $\rightarrow$ Valor percibido & $\mathrm{H}_{4}$ & $\mathrm{Y}_{13}$ & 0,54 & $6,29^{* * *}$ & Aceptada \\
\hline Imagen $\rightarrow$ Satisfacción & $\mathrm{H}_{5}$ & $\mathrm{Y}_{23}$ & 0,72 & $14,06^{\star \star \star}$ & Aceptada \\
\hline Valor percibido $\rightarrow$ Satisfacción & $\mathrm{H}_{6}$ & $\beta_{21}$ & 0,27 & $10,91^{\star \star \star}$ & Aceptada \\
\hline Valor percibido $\rightarrow$ Lealtad & $\mathrm{H}_{7}$ & $\beta_{31}$ & 0,06 & $1,79^{*}$ & Aceptada \\
\hline Satisfacción $\rightarrow$ Lealtad & $\mathrm{H}_{8}$ & $\beta_{32}$ & 0,59 & $15,98^{* * *}$ & Aceptada \\
\hline Bondad de ajuste & \multicolumn{5}{|c|}{$\begin{array}{c}\text { RMSEA }=0,06 ; \text { CFI }=0,99 ; \quad N N F I=0,98 ; \quad I F I=0,99 \\
p<0,10^{* * *} \quad p<0,01\end{array}$} \\
\hline
\end{tabular}

Fuente: Elaboración propia. 
Modelo integrado de antecedentes y consecuencias del valor percibido Sánchez F., R., Iniesta B., Má Á.; Cervera T., A. y Schlesinger D., Ma. W.

el valor percibido es también un antecedente de la lealtad del egresado, confirmándose las dos últimas hipótesis $\mathrm{H} 7$ y H8. En definitiva, todas las relaciones planteadas en el modelo, y referidas al contexto educativo, son soportadas empíricamente.

Asimismo, si se atiende a la intensidad de las relaciones, se observa cómo la imagen es la variable que ejerce una mayor influencia sobre sus consecuencias, esto es, el valor percibido y, especialmente, la satisfacción del egresado. Sin embargo, los resultados muestran la baja intensidad de la relación existente entre el valor percibido y la lealtad del egresado. Pese a que varios estudios realizados en otros contextos han soportado esta relación (por ejemplo, Sirdeshmukh et al., 2002; Yang y Peterson, 2004), los resultados obtenidos en el ámbito universitario indican que la mayor o mejor percepción de valor por parte de los egresados no es uno de los elementos que más condicionan la lealtad de los mismos a una determinada universidad, a diferencia de lo que sucede, por ejemplo, con la calidad de la interacción alumno-profesor. Dada la disparidad de los resultados obtenidos en los distintos estudios, el trabajo ha pretendido clarificar estas relaciones en la medida de lo posible, teniendo en cuenta las características del contexto educativo. Una de las razones que pueden justificar la débil relación entre el valor percibido y la lealtad del egresado es la forma en la que se ha medido el valor. Tradicionalmente, el valor ha sido definido desde una perspectiva funcional y utilitarista, donde el consumidor realiza una evaluación cognitiva de determinados atributos del producto y del establecimiento
(Dodds et al., 1991; Rust y Oliver, 1994; Zeithaml, 1988, entre otros). Frente a este planteamiento, criticado por su simplicidad y por entender el valor como algo meramente racional y económico (Mathwick et al., 2001; Sweeney y Soutar, 2001), se argumenta que cualquier experiencia de consumo o servicio puede generar tanto valor funcional como emocional (Babin y Attaway, 2000; Jones et al., 2006; Stoel et al., 2004), siendo éste un concepto complejo y suscrito a numerosas interpretaciones (Hu et al., 2009; Huber et al., 2001). Es por ello que la consideración de otro tipo de medidas de valor, donde se prime más la naturaleza emocional del concepto, o donde se considere a éste como un concepto multidimensional, pueda condicionar la naturaleza de su relación con la lealtad del egresado.

De este modo, los resultados del análisis muestran la influencia que ejerce la calidad de la interacción alumno-profesor tanto en la percepción de valor del egresado como en su lealtad hacia la institución universitaria mediante su formación a lo largo de la vida. Se completan así algunos planteamientos previos sobre la calidad de la interacción en la educación (Chung y McLarney, 2000; Plewa et al., 2005; Venkatesh y Meamber, 2006). Por otra parte, los datos obtenidos también demuestran que la confianza del egresado hacia la institución universitaria puede contribuir a la formación de su percepción de valor, aspecto que había sido soportado en otros contextos de estudio (Lentz et al., 2004; Nijssen et al., 2003; Sirdeshmukh et al., 2002). La percepción de valor también se verá incrementada en la medida en que la imagen percibida de la universidad sea mayor, según se 
había indicado ya en la literatura de servicios (Barich y Kotler, 1991; Nguyen y LeBlanc, 2001).

Del análisis de los datos obtenidos también se deriva la contribución positiva del valor percibido por el egresado a la formación de su lealtad hacia la institución universitaria. Este resultado viene a restar controversia al triángulo de relaciones valor-satisfacción-lealtad (Andreassen y Lindestad, 1998; Martín et al., 2004; Petrick et al., 2001), y a confirmar este planteamiento en el ámbito universitario. Sin embargo, los datos indican una débil relación entre el valor percibido y la lealtad, lo que puede justificarse según la forma en la que se mida el valor. Finalmente, el estudio también destaca la importancia de la imagen percibida como antecedente de la satisfacción del egresado, aspecto sobre el que no existen muchos estudios en la literatura (Mattila y Enz, 2002; Wong, 2004). En definitiva, el modelo de relaciones propuesto y los instrumentos de medida asociados al mismo pueden ser aplicados de manera fiable y válida al contexto de las instituciones de educación superior, proporcionando información de utilidad a la toma de decisiones en la gestión universitaria.

\section{Conclusiones}

La revisión de la literatura ha puesto de manifiesto que la adecuada prestación de servicios en las instituciones de educación superior se erige como una estrategia clave, tanto para el fortalecimiento institucional de las universidades y su consiguiente mejora en la calidad de la enseñanza, como para la internacionalización de sus actividades. Es, asimis- mo, un elemento para generar aportes para el desarrollo cultural y social. En consecuencia, este trabajo ha tratado de profundizar en algunas de las variables más relevantes en la experiencia de servicio en el ámbito educativo, con objeto de avanzar en una problemática de gran interés, esto es, la creación de valor en las relaciones egresado-universidad.

A través del planteamiento de un modelo de relaciones en el contexto educativo, se han analizado algunas variables clave tales como la calidad de la interacción alumno-profesor, la confianza del egresado y su percepción de la imagen y de valor de la experiencia de servicio, o la satisfacción y la lealtad del mismo. Se trata en todos los casos de instrumentos al servicio del diseño y mejora de programas formativos y metodologías docentes adecuados que permiten a las instituciones de educación superior configurarse en organizaciones de servicios innovadoras.

Se considera que el análisis empírico llevado a cabo en este estudio supone una aportación de utilidad en la investigación sobre la interacción estudiante-Universidad en la medida en que se profundiza en algunas variables y relaciones que han sido escasamente analizadas en el ámbito educativo. Más aún, las implicaciones de este estudio para la gestión de una institución pública como la Universidad son varias. En términos generales, se ha puesto de manifiesto que las instituciones universitarias han de ser conscientes de que el conocimiento, análisis y actuación sobre aspectos tales como la calidad de la interacción alumno-profesor, la imagen percibida o la confianza de los egresados van a contribuir de manera significativa al éxito en su gestión. La for- 
Modelo integrado de antecedentes y consecuencias del valor percibido Sánchez F., R., Iniesta B., $M^{a}$ Á.; Cervera T., A. y Schlesinger D., Ma. W.

mación de aspectos como la percepción de valor, la satisfacción o la lealtad del egresado a la institución educativa van a depender del éxito de la gestión de estos factores. En particular, estas instituciones desempeñan un papel crucial que debe conllevar la identificación de sus usuarios, la orientación a los mismos, la coordinación de las actividades educativas, y la consecución de los objetivos de excelencia organizativa, a través de la creación de relaciones a largo plazo basadas en el valor y la satisfacción de los egresados. Es importante tener en cuenta que hechos como el abandono prematuro de los estudios, el número de egresados que acaban el segundo ciclo de enseñanza secundaria, la falta de titulación académica y profesional de un gran número de individuos, la falta de cultura y motivación por "aprender a aprender", y las competencias clave siguen siendo elementos muy problemáticos que refuerzan la necesidad de utilizar enfoques estratégicos y de marketing que colaboren en el diagnóstico e implantación de medidas de gestión adecuadas.

En este sentido, este estudio muestra la necesidad de cuidar y potenciar la interacción alumno-profesor, en muchas ocasiones descuidada por los agentes implicados en ese proceso. Además, el conocimiento y análisis de los factores generadores de valor en los estudiantes y egresados, así como los aspectos que contribuyen a su imagen percibida y su confianza constituyen elementos de gran relevancia que deben plasmarse en los diseños curriculares de las carreras y en la gestión de las instituciones universitarias, ofreciéndose servicios de calidad que permitan al alumno egresar con las competencias esenciales para ejercer satisfactoriamente su actividad profesional en los primeros años y seguir aprendiendo de una manera continua a lo largo de su vida. En este sentido, es especialmente importante la comprensión de los elementos que generan valor en los egresados, pues puede proporcionar información de gran utilidad para la gestión de los recursos y el diseño de programas que mejor satisfagan a los estudiantes. Todos estos aspectos se encuentran entre las actuaciones que deben abordar las universidades para mejorar sus relaciones con los alumnos, y que deben contribuir a la formación de una relación de continuidad del egresado con la universidad, que le permita desarrollar una formación a lo largo de la vida adecuada y, además, vinculada principalmente a su universidad.

\section{Referencias bibliográficas}

Anderson, James C. y Gerbing, David W. (1988). Structural equation modeling in practice: A review and recommended two-step approach. Psychological Bulletin, Vol. 103, No 3, pp. 411-23.

Andreassen, Tor Wallin y Lindestad, Bodil (1998). Customer loyalty and complex services. International Journal of Service Industry Management, Vol. 9, No 1, pp. 7-23.

Arnett, D.B., German, S.D., y Hunt, S.D. (2003).The identify salience model of relationship marketing success: The case of non-profit marketing. Journal of Marketing, Vol. 67, No 4, pp. 89-105.

Babin, Barry, J. y Attaway, Jill S. (2000). Atmospheric affect as a tool for creating value and gaining share of customer. Journal of Business Research, Vol. 49, No 2, pp. 91-99. 
Babin, Barry J., Darden, William R. y Griffin, Mitch (1994). Work and/or fun: Measuring hedonic and utilitarian shopping. Journal of Consumer Research, Vol. 20, No 4, pp. 644-56.

Bagozzi, Richard P., Baumgartner, Hans y Yi, Youjae (1992). State versus action orientation and the theory of reasoned action: An aplication to coupon usage. Journal of Consumer Research, Vol. 18, No 4, pp. 505-18.

Bagozzi, Richard P. y Yi, Youjae (1988). On the evaluation of structural equation models. Journal of the Academy of Marketing Science, Vol. 16, No 1, pp. 74-94.

Baker, Julie, Parasuraman, A., Grewal, Dhruv y Voss, Glenn B. (2002). The influence of multiple store environment cues on perceived merchandise value and patronage intentions. Journal of Marketing, Vol. 66, No 2, pp. 120-41.

Baker, Stacey Menzel, Kleine, Susan Schultz y Bennion, Mark (2003). What do they know? Integration the core concept of customer value into the undergraduate marketing curriculum and its assessment. Journal of Marketing Education, Vol. 25, No 1, pp. 79-89.

Barich, H. y Kotler, Philip (1991). A framework for marketing image management. Sloan Management Review, Vol. 32, No 4, pp. 94-104.

Beerli, Asunción, Díaz, G. y Pérez, P. (2002). The configuration of the university image and its relationship with the satisfaction of students. Journal of Educational Administration, Vol. 40, No 5, pp. 496-505.

Bergami, M. y Bagozzi, Richard P. (2000). Self-categorization, affective commitment and group self-esteem as distinct aspects of social identity in the organization. British Journal of Social Psychology, Vol. 39, No 4, pp. 55577.
Bhattacharya, C. y Sen, S. (2003). Consumer company identification: A framework for understaning consumers' relationships with companies. Journal of Marketing, Vol. 67, p. 2.

Bloemer, J. y De Ruyter, Ko (1998). On the relationship between store image, store satisfaction and store loyalty. European Journal of Marketing, Vol. 32, No 5/6, pp. 499-513.

Bloemer, J.; De Ruyter, Ko y Peeters, P. (1998). Investigating drivers of bank loyalty: the complex relationship between image, service quality and satisfaction. International Journal of Bank Marketing, Vol. 16, No 7, pp. 276-86.

Bolton, Ruth N. y Drew, James H. (1991). A multistage model of customers' assessments of service quality and value. Journal of Consumer Research, Vol. 17, No 4, pp. 375-84.

Bryce, H. (2007). The public's trust in nonprofit organizations: The role of relationship marketing and management. California Management Review, Vol. 49, No 4, pp. 112-32.

Caruana, Albert, Money, Arthur H., y Berthon, Pierre R. (2000). Service quality and satisfaction - the moderating role of value. European Journal of Marketing, Vol. 34, No 11/12, pp. 1338-52.

Cave, M., Hannery, S., Henkel, M., y Fogan, M. (1997). The use of performance indicators in higher education: a critical analysis of developing practice. Jessica Kingsley Publishers, London and Bristol, Pennsylvania.

Cronin, J. Joseph, Jr., Brady, Michael K., Brand, Richard R., Hightower, Roscoe, Jr., y Shemwell, Donald J. (1997). A cross-sectional test of the effect and conceptualization of service value. The Journal of Services Marketing, Vol. 11, No 6, pp. 375-91. 
Modelo integrado de antecedentes y consecuencias del valor percibido Sánchez F., R., Iniesta B., $M^{a}$ Á.; Cervera T., A. y Schlesinger D., Ma. W.

Cronin, J. Joseph; Jr., Brady, Michael K. y Hult, G. Tomas M. (2000). Assessing the effects of quality, value, and customer satisfaction on consumer behavioral intentions in service environments. Journal of Retailing, Vol. 76, No 2, pp 193-218.

Crosby, L.A.; Evans, K.R. y Cowles, D. (1990). Relationship quality in services selling: an interpersonal influence perspective. Journal of Marketing, Vol. 54, No 3, pp 68-81.

Chang, Tung-Zong y Wildt, Albert R. (1994). Price, product information, and purchase intention: An empirical study. Journal of the Academy of Marketing Science, Vol. 22, No 1, pp 16-27.

Chen, Zhan y Dubinsky, Alan J. (2003). A conceptual model of perceived customer value in e-commerce: A preliminary investigation. Psychology \& Marketing, Vol. 20, No 4, pp 323-47.

Cheng, Y.C. y Tam, W.M. (1997). Multi-models of quality in education. Quality Assurance in Education, Vol. 5, No 1, pp. 22-31.

Chung, E. y McLarney, C. (2000). The classroom as a service encounter: Suggestions for value creation. Journal of Marketing Education, Vol. 24, No 4, pp. 484-500.

Day, Ellen (2002), The role of value in consumer satisfaction. Journal of Consumer Satisfaction, Dissatisfaction and Complaining Behavior, Vol. 15, No 22-32.

Declaración de Bolognia (1999). European space for higher education. European Commision, 19 de junio.

De Ruyter, Ko, Wetzels, Martin, Lemmink, Jos, y Mattsson, Jan (1997). The dynamics of the service delivery process: A value-based approach. International Journal of Research in Marketing, Vol. 14, No 3, pp. 231-43.
Dick, Alan S. y Basu, Kunal (1994). Customer loyalty: Toward an integrated conceptual framework. Journal of the Academy of Marketing Science, Vol. 22, No 2, pp. 99-113.

Dodds, William B. y Monroe, Kent B. (1985). The effect of brand and price information on subjetive product evaluations. Advances in Consumer Research, Vol. 12, No 1, pp. 85-90.

Dodds, William B., Monroe, Kent B. y Grewal, Dhruv (1991). Effects of price, brand, and store information on buyers' product evaluations. Journal of Marketing Research, Vol. 28, No 3, pp. 307-19.

Eggert, Andreas y Ulaga, Wolfgang (2002). Customer perceived value: A substitute for satisfaction in business markets?. The Journal of Business \& Industrial Marketing, Vol. 17, No 2/3, pp. 107-18.

Elliot, K.M. y Healy, M.A. (2001). Key factors influencing student satisfaction related to recruitment and retention. Journal of Marketing for Higher Education, Vol. 10, No 4, pp. 1-11.

Flavián, C. y Lozano, F.J. (2001). Orientación al mercado y resultados: Una aplicación en el sector educativo español. XIII Encuentro de Profesores Universitarios de Marketing, Málaga.

Fornell, Claes (1992). A national customer satisfaction barometer, the Swedish experience. Journal of Marketing, Vol. 56, No 1, pp. 6-21.

Fornell, Claes y Larcker, David F. (1981). Evaluating structural equation models with unobservable variables and measurement error. Journal of Marketing Research, Vol. 18, No 1, pp. 39-50.

Gil, Irene; Sánchez, Manuel; Berenguer, Gloria y González-Gallarza, Martina (2005). Encuentro de servicio, valor percibido y satisfacción del cliente en la relación 
entre empresas. Cuadernos de Estudios Empresariales, Vol. 15, No 47-72.

Gwinner, K.; Gremler, D. y Bitner, Mary Jo (1998). Relational benefits in services industries: the customer's perspective. Journal of the Academy of Marketing Science, Vol. 26, No 2, pp. 101-14.

Helgesen, O. y Nesset, E. (2007). What accounts for students' loyalty? Some field study evidence. International Journal of Educational Management, Vol. 21, No 2, pp. 126-43.

Henning-Thurau, T. y Klee, A. (1997). The impact of customer satisfaction and relationship quality on customer retention: a critical reassessment and model development. Psychology \& Marketing, Vol. 14, No 8, pp. 737-65.

Henning-Thurau, T.; Langer, M.F. y Hansen, U. (2001). Modelling and managing student loyalty. An approach based on the concept of relationship quality. Journal of Service Research, Vol. 3, No 4, pp. 331-44.

Holbrook, Morris B. (1999). Introduction to consumer value. En Consumer value. A framework for analysis and research. Routledge.

Holbrook, Morris B. y Corfman, Kim P. (1985). Quality and value in the consumption experience: Phaedrus rides again. En Perceived quality: How consumers view stores and merchandise. Lexington Books.

Holbrook, Morris B. y Hirschman, Elizabeth C. (1982). The experiential aspects of consumption: Consumer fantasies, feelings, and fun. Journal of Consumer Research, Vol. 9, No 2, pp. $132-40$.

Holdford, D. y White, S. (1997). Testing commitment-trust theory in relationships between pharmacy schools and students. American Journal of Phar- maceutical Education, Vol. 61, No 249-56.

Hu, Hsin-Hui; Kandampully, Jay y Juwaheer, Thanika Devi (2009). Relationships and impacts of service quality, perceived value, customer satisfaction, and image: An empirical study. The Service Industries Journal, Vol. 29, No 2, pp. 111-25.

Huber, Frank; Herrmann, Andreas y Hennenberg, Stephan C. (2007). Measuring customer value and satisfaction in services transactions, scale development, validation and cross-culturan comparison. International Journal of Consumer Studies, Vol. 31, No 554-64.

Huber, Frank; Herrmann, Andreas y Morgan, Robert E. (2001). Gaining competitive advantage through customer value oriented management. The Journal of Consumer Marketing, Vol. 18, No 1, pp. 41-53.

Ivy, J. (2001). Higher education institution image: a correspondence analysis approach. International Journal of Educational Management, Vol. 15, No 6/7, pp. 276-82.

Javalgi, Rajshekhar G.; Joseph, W. Benoy y LaRosa, Richard J. (2009). Crosscultural marketing strategies for delivering knowledge-based services in a borderless word: The case of management education. Journal of Services Marketing, Vol. 23, No 6, pp. 371-84

Jones, Michael A.; Reynolds, Kristy E. y Arnold, Mark J. (2006). Hedonic and utilitarian shopping value: Investigating differential effects on retail outcomes. Journal of Business Research, Vol. 59, No 9, pp. 974-81.

Keng, Ching-Jui; Huang, Tseng-Lung; Zheng, Li-Jie y Hsu, Maxwell, K. (2007). Modeling service encounters and customer experiential value in retailing. 
Modelo integrado de antecedentes y consecuencias del valor percibido Sánchez F., R., Iniesta B., $M^{a}$ Á.; Cervera T., A. y Schlesinger D., Ma. W.

An empirical investigation of shopping mall customers in Taiwan. International Journal of Service Industry Management, Vol. 18, No 4, pp. 34967.

Kotler, Philip y Fox, K. (1995). Strategic marketing for educational institutions 2nd Edition. Prentice Hall, Englewood Cliffs, NJ.

Krehbiel, T.; McClure, R. y Pratsini, E. (1997). Using student disconfirmation as a measure of classroom effectiveness. Journal of Education for Business, Vol. 72, No 4, pp. 224-30.

Landrum, R.E., Turrisi, R. y Harless, C. (1998). University image: the benefits of assessment and modelling. Journal of Marketing for Higher Education, Vol. 9, No 1, pp. 53-68.

Lentz, P.; Teusner, S. y Holzmueller, H.H. (2004). Customer satisfaction, trust, value, and loyalty in relational B2B exchanges. EMAC Conference, Murcia, Spain.

Lindgreen, Adam y Wynstra, Finn (2005). Value in business markets: What do we know? Where are we going?. Industrial Marketing Management, Vol. 34, No 7, pp. 732-48.

Martensen, A.; Gronholdt, L.; Eskildsen, J. y Kristensen, K. (1999). Measuring student oriented quality in higher education: application of the ECSI methodology, TQM for Higher Education Institutions, Verona.

Martín, David; Barroso, Carmen y Martín, Enrique (2004). El valor percibido de un servicio. Revista Española de Investigación de Marketing ESIC, Vol. 8, No 1, pp. 47-73.

Mathwick, Charla; Malhotra, Naresh y Rigdon, Edward (2001). Experiential value: Conceptualization, measurement and application in the catalog and Internet shopping environment. Journal of Retailing, Vol. 77, No 1, pp. 39-56.
Mattila, A. y Enz, C. (2002). The role of emotions in service encounters. Journal of Service Research, Vol. 4, No 4, pp. 268-77.

Morgan, R. y Hunt, S. (1994). The Commitment Trust Theory of Relationship Marketing. Journal of Marketing, Vol. 58, No 3, pp. 20-38.

Nguyen, Nha y LeBlanc, Gaston (2001). Image and reputation of higher education institutions in students' retention decisions. The International Journal of Educational Management, Vol. 15, No 6, pp. 303-11.

Nijssen, Ed; Singh, Jagdip; Sirdeshmukh, Deepak y Holzmüeller, Hartmut (2003). Investigating industriy context effects in consumer-firm relationships: Preliminary results from a dispositional approach. Journal of the Academy of Marketing Science, Vol. 31, No 1, pp. $46-60$.

Nunnally, Jum C. (1978). Psychometric theory, McGraw-Hill, New York.

Oliver, Richard L. (1999). Value as excellence in the consumption experience. En Consumer value. A framework for analysis and research. Routledge.

Oplatka, I. y Hemsley-Brown, J. (2004). The research on school marketing: Current issues, future directions. Journal of Educational Administration, Vol. 42, No 3, pp. 375-400.

Paramewaran, R. y Glowacka, A.E. (1995). University image: an information processing perspective. Journal of Marketing for Higher Education, Vol. 6, No 2, pp. 41-56.

Parvatiyar, A. y Sheth, J.N. (1994). Paradigm shift in marketing theory and approach: The emergence of relationship marketing. En Relationship Marketing: Theory, Methods and Applications. Emory University.

Patterson, Paul G. y Spreng, Richard A. (1997). Modelling the relationship be- 
tween perceived value, satisfaction and repurchase intentions in a business-to-business, services context: an empirical examination. International Journal of Service Industry Management, Vol. 8, No 5, pp. 414-34.

Peiró, J.M.; Martínez-Tur, V.; y Ramos, J. (2005). Employees' overestimation of functional and relational service quality: A gap analysis. The Service Industries Journal, Vol. 25, No 6, pp. 1-17.

Petrick, James F.; Morais, Duarte D. y Norman, William C. (2001). An examination of the determinants of entertainment vacationers' intentions to revisit. Journal of Travel Research, Vol. 40, No 1, pp. 41-48.

Pina, J.; Martínez, E. De Chernatony, Leslie, y Drury, S. (2006). Th effect of service brand extensions on corporate image. An empirical model. European Journal of Marketing, Vol. 40, No 1/2, pp. 174-97.

Plewa, C. y Quester, Pascale G. (2006). Satisfaction with university-industry relationships: the impact of commitment, trust and championship. International Journal of Technology Transfer \& Commercialisation, Vol. 5, No 1/2, pp 79-101.

Plewa, C.; Quester, Pascale G. y Baaken, T. (2005). Relationship marketing and university-industry linkages: A conceptual framework. Marketing Theory, Vol. 5, No 4, pp. 433-56.

Pollak, Birgit Leisen (2009). Linking the hierarchical service quality model to customer satisfaction and loyalty. Journal of Services Marketing, Vol. 23, No 1, pp. 42-50.

Porter, M. (1985), Competitive Advantage. The Free Press, New York

Price, L.L.; Amould, E.J. y Tierney, P. (1995). Going to extremes: managing service encounters and assessing provider performance. Journal of Marketing, Vol. 59, No 2, pp. 83-97.

Pura, Minna (2005). Linking perceived value and loyalty in location-based mobile services. Managing Service Quality, Vol. 15, No 6, pp. 509-38.

Rapp, R. (2000). Customer relationship marketing in the airline industry. En Relationship marketing: Gaining competitive advantage through customer satisfaction and customer retention, Springer.

Roberts, K.; Varki, Sajeev y Brodie, R. (2003). Measuring the quality of relationships in consumer services: an empirical study. European Journal of Marketing, Vol. 37, No 1/2, pp. 169-96.

Ruiz de Maya, Salvador (2001). El comportamiento del consumidor en marketing. Del método científico a su posición en la empresa. Cuadernos de Administración, Vol. 26, No 35-57.

Rust, Roland T. y Oliver, Richard L. (1994). Service quality: Insights and managerial implications from the frontier. En Service quality: New directions in theory and practice. Sage Publications.

Sakthivel, P.B. y Raju, R. (2006). An instrument for measuring engineering education quality from students' perspective. The Quality Management Journal, Vol. 13, No 3, pp. 23-34.

Sánchez, Manuel, Gil, Irene, y Mollá, Alejandro (2001). Estatus actual del marketing de relaciones. Revista Europea de Dirección y Economía de la Empresa, Vol. 9, No 3, pp. 47-64.

Saxe, R. y Weitz, B.A. (1982). The SOcO scale: A measure of customer orientation of salespeople. Journal of Marketing Research, Vol. 19, No 3, pp. 343-51. 
Modelo integrado de antecedentes y consecuencias del valor percibido

Sánchez F., R., Iniesta B., $M^{a}$ Á.; Cervera T., A. y Schlesinger D., Ma. W.

Sebastián, J. (2004). Cooperación e internacionalización de las universidades. Editorial Biblos, Argentina.

Sheth, Jagdish N., Newman, Bruce I., y Gross, Barbara L. (1991). Consumption values and market choices. Theory and applications. South-Western Publishing Co. Cincinnati.

Sirdeshmukh, Deepak., Singh, Jagdip., y Sabol, Barry (2002). Consumer trust, value, and loyalty in relational exchanges. Journal of Marketing, Vol. 66 , No 1, pp. 15-37.

Söderlund, Magnus (2006). Measuring customer loyalty with multi-item scales: A case for caution. International Journal of Service Industry Management, Vol. 17, No 1, pp. 76-98.

Stauss, B. y Neuhaus, P. (1997). The qualitative satisfaction model. International Journal of Service Industry Management, Vol. 8, No 3/4, pp. 236-49.

Stoel, Leslie, Wickliffe, Vanessa, y Lee, Kyu Hye (2004). Attribute beliefs and spending as antecedents to shopping value. Journal of Business Research, Vol. 57, No 10, pp. 1067-73.

Sweeney, Jillian C. y Soutar, Geoffrey N. (2001). Consumer perceived value: The development of a multiple item scale. Journal of Retailing, Vol. 77, No 2, pp. 203-20.

Tam, Jackie L.M. (2004). Customer satisfaction, service quality and perceived value: An integrative model. Journal of Marketing Management, Vol. 20, No 7,8, pp. 897-917.

Unni, R.M. (2005). Value perceptions and retention of textbooks among marketing and other business majors. Marketing Education Review, Vol. 15, No 2, pp. 71-79.

Varki, Sajeev y Colgate, Mark (2001). The role of price perceptions in an integrated model of behavioral intentions. Jour- nal of Service Research, Vol. 3, No 3, pp. 232-40.

Venkatesh, Alladi y Meamber, Laurie A. (2006). Arts and aesthetics: Marketing and cultural production. Marketing Theory, Vol. 6, No 1, pp. 11-39.

Vesel, Patrick y Zabkar, Vesna (2010). Relationship quality evaluation in retailers' relationships with consumers. European Journal of Marketing, Vol. 44, No 9/10, pp. 1334-65.

Wang, Yonggui; Lo, Hing Po; Chi, Renyong y Yang, Yongheng (2004). An integrated framework for customer value and customer-relationship-management performance: A customer-based perspective from China. Managing Service Quality, Vol. 14, No 2/3, pp. 169-82.

Webster, F. E. Jr. (1994). Defining the new marketing concept. Marketing Management, Vol. 2, No 4, pp. 22-31.

Webster, Robert L. y Hammond, Kevin L. (2008). Does a market orientation strategy exist toward business school students? A view from three levels of academic administrators. Academy of Marketing Studies Journal, Vol. 12, No 2, pp. 19-33.

Wetzels, Martin, De Ruyter, Ko, y Van Birgelen, M.V. (1998). Marketing service relationships: The role of commitment. Journal of Business \& Industrial Marketing, Vol. 13, No 4/5, pp. 40623.

Wong, A. (2004). The role of emotional satisfaction in service encounters. Managing Service Quality, Vol. 14, No 5, pp. 365-76.

Wong, E. (2005). Operationalization of strategic change in continuing education. The International Journal of Educational Management, Vol. 19, No 5, pp. 383-96.

Woodruff, Robert B. (1997). Customer value: The next source for competitive advantage. Journal of the Academy of 
Marketing Science, Vol. 25, No 2, pp. 139-53.

Woodruff, Robert B. y Gardial, Sarah F. (1996). Know your customer. New approaches to understanding customer value and satisfaction. Blackwell Publishers, Inc., Cambridge.

Yang, Zhilin y Peterson, Robin T. (2004). Customer perceived value, satisfaction, and loyalty: The role of switching costs. Psychology \& Marketing, Vol. 21, No 10, pp. 799-822.

Yavas, Ugur y Babakus, Emin (2009). Retail store loyalty: A comparison of two customer segments. International Journal of Retail \& Distribution Management, Vol. 37, No 6, pp. 477-92.

Zeithaml, Valarie A. (1988). Consumer perceptions of price, quality, and value: a means-end model and synthesis of evidence. Journal of Marketing, Vol. 52, No 3, pp. 2-22. 